\title{
Heterotopia de mucosa gástrica en el intestino delgado, hallazgo en videocápsula endoscópica. Reporte de caso
}

\section{An endoscopic videocapsule finding of heterotopia of the gastric mucosa of the small intestine}

\author{
Santiago Castaño Quintero, ${ }^{1 *}$ Natalia Calvache, ${ }^{2}$ Mauricio Sepúlveda, ${ }^{3}$ Catalina Maldonado, ${ }^{3}$ Pedro Tomás Argüello, ${ }^{4}$ Juliana Escobar, ${ }^{5}$ \\ Carlos Arturo Rojas. ${ }^{3}$
}

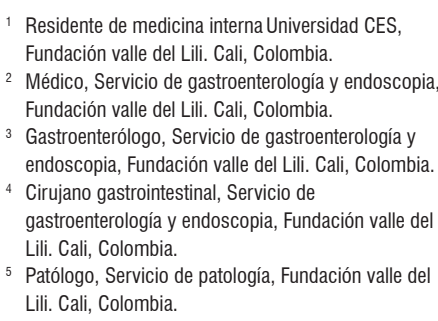

5 Patólogo, Servicio de patología, Fundación valle del Lili. Cali, Colombia.

*Correspondencia: santiagocasta1989@gmail.com.

\begin{abstract}
Resumen
Introducción: la heterotopia de mucosa gástrica se refiere a la localización ectópica de mucosa gástrica en cualquier parte del tracto gastrointestinal. Es una causa poco frecuente de úlceras gastrointestinales y sangrado digestivo oculto. La videocápsula endoscópica se ha convertido en una herramienta fundamental para el estudio del intestino delgado. Métodos: estudio descriptivo de reporte de caso. La información de la historia clínica, reporte de patología y estudios endoscópicos, se extrajo de las bases de datos de la Fundación Valle del Lili. Resultados: paciente masculino de 71 años, en quien se realizó videocápsula endoscópica por un sangrado digestivo oculto y se encontraron segmentos de estenosis y úlceras en el yeyuno. La histopatología reveló la presencia de mucosa gástrica heterotópica. Conclusión: la heterotopia de mucosa gástrica debe considerarse como una causa posible de sangrado del intestino delgado.
\end{abstract}

\section{Palabras clave}

Heterotopia gástrica, sangrado digestivo oculto, videocápsula endoscópica.

\begin{abstract}
Introduction: Heterotopia of the gastric mucosa refers to an ectopic location of gastric mucosa in any part of the gastrointestinal tract. It is a rare cause of gastrointestinal ulcers and occult digestive bleeding. Endoscopic videocapsules have become fundamental tools for study of the small intestine. Methods: This is a descriptive case study based on information from the medical history, pathology report and endoscopic studies extracted from the databases of the Fundación Valle del Lili in Cali, Colombia. Results: An endoscopic videocapsule was used to examine a 71-year-old male patient who suffered from occult digestive bleeding. Segments of stenosis and ulcers were found in the jejunum and histopathology revealed heterotopic gastric mucosa. Conclusion: Heterotopy of the gastric mucosa should be thought of as a possible cause of bleeding in the small intestine.
\end{abstract}

Keywords

Gastric heterotopy, occult digestive bleeding, endoscopic videocapsule.

\section{INTRODUCCIÓN}

La presencia de úlceras en el intestino delgado es una condición poco común. La llegada de técnicas endoscópicas como la videocápsula y la enteroscopia han permitido identificar con mayor frecuencia este tipo de lesiones. Las úlceras intestinales se pueden manifestar clínicamente con anemia, episodios de dolor abdominal, hemorragia, obstrucción o perforación (1). La heterotopia de mucosa gástrica se refiere a la presencia de mucosa gástrica en una localización anormal; pocas veces se encuentra por debajo del ligamento de Treitz, lo que puede causar úlceras intes- 
tinales. Es difícil diferenciar la heterotopia de la metaplasia gástrica, la cual se asocia con enfermedad de Crohn (2).

Se presenta el caso de un paciente masculino en cuya videocápsula por estudio de sangrado digestivo se documentó la presencia de múltiples úlceras en el intestino delgado que condicionaban a estenosis en varios segmentos. El estudio histológico reveló la presencia de glándulas pilóricas con epitelio foveolar gástrico.

\section{CASO CLÍNICO}

Es un paciente masculino de 71 años, con antecedente de hipertensión arterial y enfermedad diverticular. Asistió a la unidad de endoscopia de la Fundación Valle del Lili para la realización de videocápsula de manera ambulatoria. Presentaba un cuadro clínico de 2 años de evolución de dolor abdominal, cólico y sensación de masa en la fosa ilíaca derecha, asociado con distensión abdominal y episodios de constipación que se alternaban con deposiciones diarreicas acuosas ocasionales. En los estudios paraclínicos se había documentado sangre oculta en heces positiva y anemia ferropénica refractaria al uso de sulfato ferroso. Negaba el consumo de antiinflamatorios no esteroideos (AINE), a los cuales mencionaba ser alérgico. Traía reportes de endoscopia de vías digestivas altas (EVDA) y colonoscopia total extrainstitucionales que eran normales. Se consideró que se trataba de un sangrado digestivo cuyo origen probable era el intestino delgado.

Se inició el estudio con videocápsula, previa preparación y consentimiento informado. En el yeyuno proximal se observaron erosiones y pequeñas úlceras aisladas; a nivel del yeyuno medio habían erosiones, úlceras profundas de aspecto crónico con fibrina y retracciones de pliegues con mucosa edematizada alrededor, lo cual condicionaba a segmentos de estenosis parciales. La cápsula fue retenida por una lesión estenótica ulcerada en el yeyuno distal (Figura 1).
Una semana después del estudio, consultó por un cuadro de obstrucción intestinal, por lo que se realizó una fluoroscopia en la que se documentó la persistencia de la cápsula en el cuadrante inferior izquierdo del intestino delgado. Se llevó a laparoscopia exploratoria, en la que se reportó una brida del epiplón a nivel del íleon distal que generaba una obstrucción parcial del intestino delgado. Distal a la obstrucción, había dilatación del asa con serosa engrosada y congestiva. La cápsula estaba retenida en el segmento estenótico, lo cual requirió una resección de $6,5 \mathrm{~cm}$ de intestino (Figura 2).

La pieza quirúrgica se llevó a estudio anatomopatológico, en el que se reportaron a nivel macroscópico dos lesiones ulceradas $(1,5 \mathrm{~cm}$ x $1 \mathrm{~cm}$ y $1 \mathrm{~cm}$ x $1 \mathrm{~cm})$. El examen microscópico mostró el intestino delgado con áreas de ulceración de la mucosa donde había tejido de granulación y depósitos de fibrina. Se encontraron focos en los que se observaba epitelio gástrico foveolar y, a nivel de la región de la mucosa-submucosa, unas glándulas pilóricas sin atipia citológica. Se observó un infiltrado inflamatorio linfoplasmocitario con formación de nódulos linfoides hiperplásicos. Los márgenes quirúrgicos se apreciaron con adecuada viabilidad. Se realizó histoquímica de PAS (periodic acidSchiff) e inmunohistoquímica con el marcador MUC-5AC para mucinas, y se interpretó como la presencia de mucinas neutras en la mucosa gástrica representada por el epitelio foveolar y las glándulas pilóricas (Figura 3).

Se realizó un control postoperatorio por consulta externa, en el que se inició el manejo con omeprazol. En citas posteriores, se documentó mejoría de los síntomas gastrointestinales y resolución del cuadro anémico. Se realizaron estudios complementarios con anticuerpos anticitoplasma de neutrófilos (ANCA), anticuerpos anti-sacharomyces y factor reumatoideo negativos; velocidad de eritrosedimentación y proteína C-reactiva (PCR) cualitativa positivos. Se decidió continuar el seguimiento clínico y con exámenes de laboratorio trimestrales.
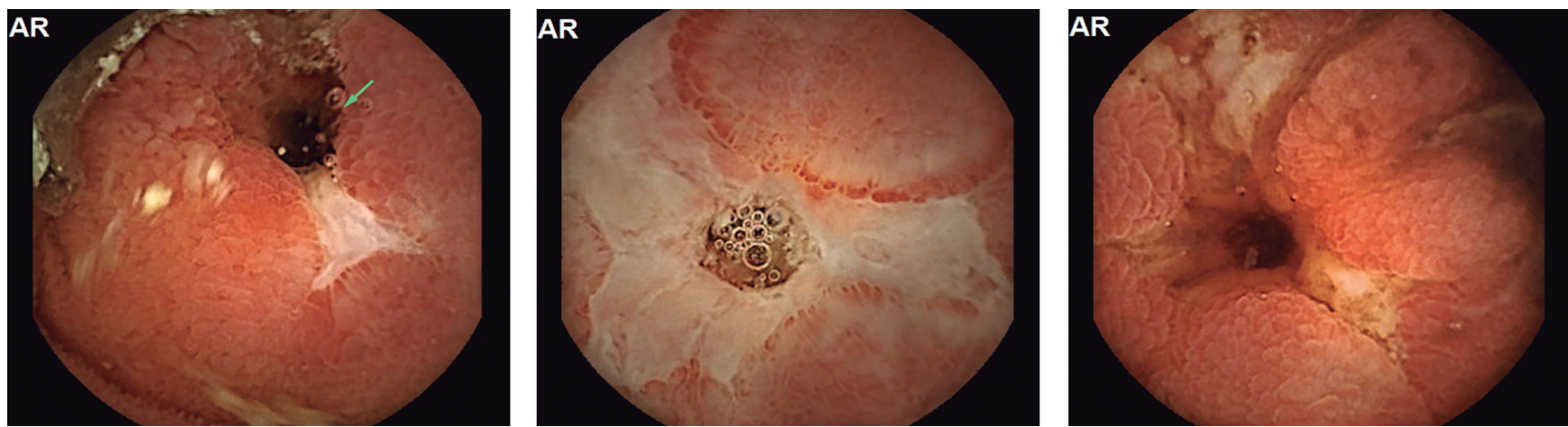

Figura 1. Videocápsula endoscópica. Estenosis ulcerada concéntrica, rodeada de mucosa edematizada. 


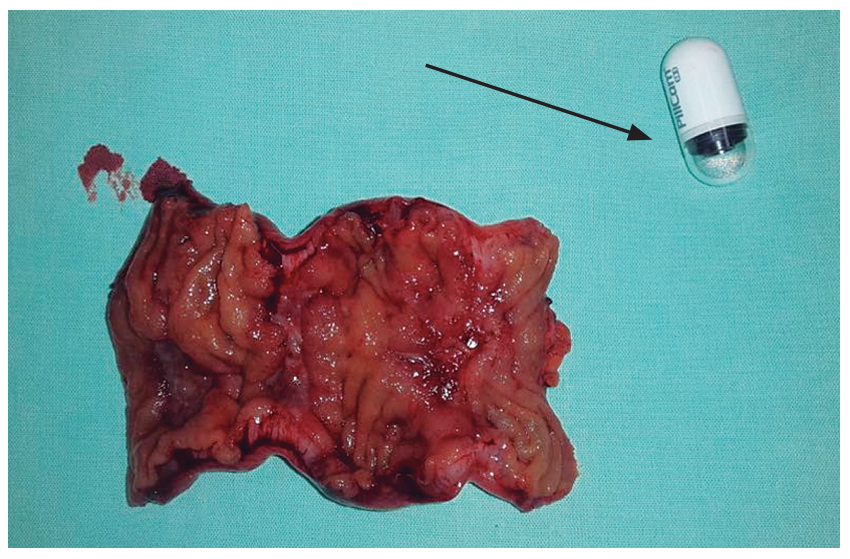

Figura 2. Pieza quirúrgica. Segmento estenótico de $6,5 \mathrm{~cm}$ de intestino delgado (íleon). Videocápsula endoscópica (flecha).

\section{DISCUSIÓN}

El desarrollo y uso de la videocápsula endoscópica ha posibilitado el diagnóstico de diferentes lesiones en el intestino delgado que previamente eran difíciles de identificar (3). Este estudio se ha convertido en una herramienta primordial en la valoración de lesiones hemorrágicas, úlceras y tumores en este órgano (4). Actualmente es considerado un estudio de primera línea en el enfoque del antes llamado sangrado digestivo oscuro, término que cambió a posible sangrado del intestino delgado y que ocurre en el $5 \%-10 \%$ de los pacientes que consultan por sangrado gastrointestinal (5-7).

En un estudio realizado por Pandey y colaboradores sobre los pacientes sometidos a videocápsula endoscópica, se demostró que las causas más frecuentes de sangrado con
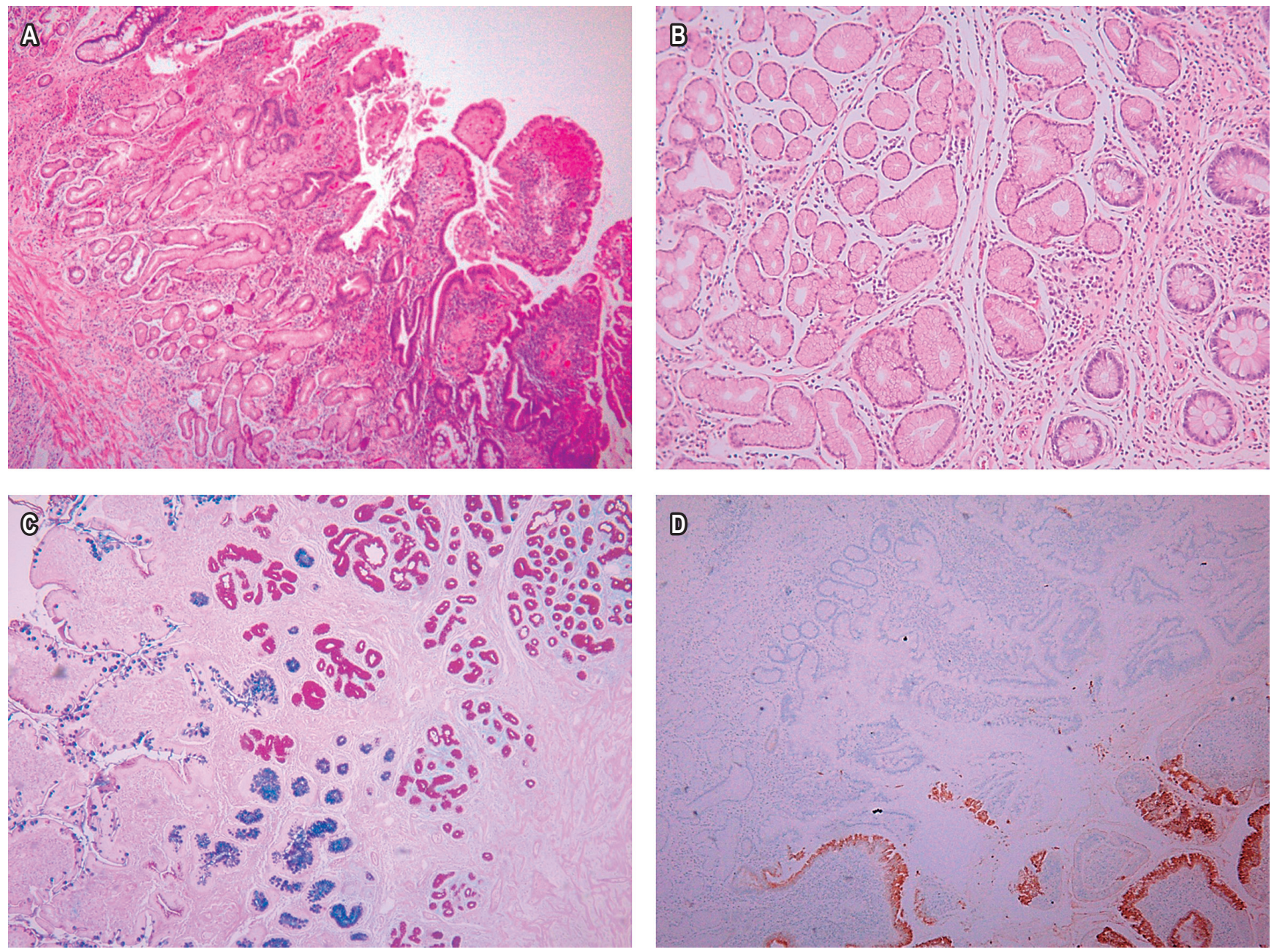

Figura 3. Estudio histopatológico. A. Se observan zonas revestidas por el epitelio gástrico foveolar (H-E x 10). B. Glándulas pilóricas en la mucosa y submucosa (H-E x 40). C y D. Tinción con PAS y estudio inmunohistoquímico con MUC-5AC que muestran la presencia de mucinas neutras de la mucosa gástrica representadas por el epitelio foveolar y las glándulas pilóricas. 
origen en el intestino delgado fueron la angiodisplasia, la cual se presentó hasta en un $23,5 \%$, seguido de enfermedad de Crohn en el 14,7\% y la enteropatía AINE en el 11,76\%. Las úlceras en el intestino delgado como causa de sangrado son infrecuentes, se han reportado hasta en un 5,8 \% (8). Estas úlceras son, por lo general, secundarias a enfermedad de Crohn o enteropatía por AINE, y con menor frecuencia se asocian con entidades como la enfermedad celíaca, enfermedad de Behçet, yeyunoileítis no especificada, vasculitis, tumores estromales gastrointestinales, adenocarcinoma intestinal, linfoma, mieloma múltiple, divertículo de Meckel, infecciones y heterotopia gástrica (9).

La heterotopia de la mucosa gástrica se refiere a la localización ectópica de mucosa gástrica en cualquier parte del tracto gastrointestinal. El esófago es el sitio en el que con mayor frecuencia se identifican este tipo de lesiones, seguido por el duodeno (10-12). Con excepción de la heterotopia gástrica ubicada en un divertículo de Meckel, encontrarla por debajo del ligamento de Treitz es extremadamente raro. Se han reportado pocos casos en la literatura médica de heterotopia gástrica en el yeyuno, íleon, colon y recto $(13,14)$. En la mayoría de los reportes que involucran el intestino delgado, los pacientes suelen presentarse asintomáticos y los hallazgos se identifican por medio de estudios histopatológicos de muestras obtenidas vía quirúrgica. Sin embargo, se han descrito síntomas asociados como anemia, sangrado gastrointestinal $(15,16)$, intususcepción (17), ulceración, perforación y obstrucción intestinal; además, hay casos de trasformación a malignidad (14, 18-20). En nuestro paciente, los síntomas fueron dolor abdominal $\mathrm{y}$ anemia con un posterior cuadro de obstrucción intestinal luego de la retención de la videocápsula endoscópica. Los hallazgos de afección multifocal del intestino delgado por presencia de varias úlceras que conducían a diferentes segmentos de estenosis es una situación muy poco común. Existen solo dos casos similares reportados en la literatura, uno en una paciente de 42 años con episodios de dolor abdominal recurrente y el segundo en un paciente de 24 años con un cuadro de dolor abdominal y perforación intestinal $(2,21)$. Además, solo en un caso las lesiones se identificaron por medio de videocápsula endoscópica (14).

En relación con los hallazgos histológicos, se han caracterizado dos tipos de heterotopia gástrica. El primero, en el que se evidencia tanto glándulas gástricas como epitelio foveolar gástrico; situación presente en nuestro paciente. $\mathrm{Y}$ el segundo, en el que solo se encuentra epitelio foveolar gástrico. Se cree que el primero tiene su origen en una anormalidad congénita del tracto gastrointestinal, mientras que el segundo podría ser adquirido por medio de un proceso metaplásico, cuyo mecanismo continúa siendo desconocido $(11,15,22)$. Existe la posibilidad de que este tipo de lesiones histológicas estén relacionadas con enfermedad de
Crohn; se han identificado focos de metaplasia pilórica en pacientes con úlceras en el íleon y esta enfermedad (23). Koukoulis y colaboradores evaluaron 45 biopsias del íleon terminal de diferentes pacientes e identificaron metaplasia de glándulas pilóricas en 10 (22,2 \%) especímenes. Estos hallazgos se observaron en pacientes con ileítis terminal y se localizaron por debajo de criptas de regeneración, cerca al tejido de granulación y ulceración; en ninguna de las piezas se observaron granulomas. El significado de este tipo de lesiones no es del todo claro y se ha considerado un hallazgo inespecífico (24). Agarwal y colaboradores, en un estudio retrospectivo, identificaron que el hallazgo de metaplasia pilórica de pacientes con enfermedad inflamatoria intestinal en quienes se realizó anastomosis ileoanal con bolsa ileal favorecía el diagnóstico de enfermedad de Crohn sobre colitis ulcerativa; además, se asoció con complicaciones postoperatorias como la pouchitis crónica (25). A pesar de que en el caso de nuestro paciente no existe evidencia suficiente que sugiera la presencia de enfermedad de Crohn, esta posibilidad no puede descartarse por completo, lo que hace que el seguimiento clínico, paraclínico e imagenológico sean necesario.

\section{CONCLUSIÓN}

La videocápsula endoscópica ha hecho posible la obtención de imágenes del intestino delgado, antes no identificadas. La heterotopia de mucosa gástrica es una condición que tradicionalmente se ha considerado congénita y que muy rara vez compromete el tracto gastrointestinal por debajo del ligamento de Treitz. Es muy poco frecuente que presente una afección multifocal en el intestino delgado, como en el caso de este paciente. Distinguir la heterotopia gástrica de la metaplasia pilórica es difícil y aún sigue siendo un punto de discusión. Esta situación obliga a que los pacientes diagnosticados con esta afección requieran un seguimiento clínico, paraclínico e imagenológico estricto.

\section{REFERENCIAS}

1. Fisher L, Scheiman JM. Intestinal Ulcerations. En: Feldman M, Friedman LS, Brandt LJ (editores). Sleisenger and Fordran's gastrointestinal and liver disease. 10. ${ }^{\mathrm{a}}$ edición. Filadelfia: Saunders; 2016. p. 2102-11.

2. Vani M, Nambiar A, Geetha K, Kundil B. Jejunal Gastric Heterotopia causing Multiple Strictures and Perforation Peritonitis- A Case Report with Review of Literature. J Clin Diagn Res. 2017;11(3):ED11-ED12. https://doi. org/10.7860/JCDR/2017/25585.9590.

3. Micic D, Semrad CE. Small Bowel Endoscopy. Curr Treat Options Gastroenterol. 2016;14(2):220-35. https://doi. org/10.1007/s11938-016-0095-x. 
4. Ching HL, McAlindon ME, Sidhu R. An update on small bowel endoscopy. Curr Opin Gastroenterol. 2017;33(3):181-8. https://doi.org/10.1097/MOG. 0000000000000346.

5. Gerson LB, Fidler JL, Cave DR, Leighton JA. ACG Clinical Guideline: Diagnosis and Management of Small Bowel Bleeding. Am J Gastroenterol. 2015;110(9):1265-87. https://doi.org/10.1038/ajg.2015.246.

6. Pennazio M, Spada C, Eliakim R, Keuchel M, May A, Mulder CJ, et al. Small-bowel capsule endoscopy and device-assisted enteroscopy for diagnosis and treatment of small-bowel disorders: European Society of Gastrointestinal Endoscopy (ESGE) Clinical Guideline. Endoscopy. 2015;47(4):35276. https://doi.org/10.1055/s-0034-1391855.

7. Gerson LB. Small Bowel Bleeding: Updated Algorithm and Outcomes. Gastrointest Endoscopy Clin N Am. 2017;27(1):171-80. https://doi.org/10.1016/j.giec.2016. 08.010 .

8. Pandey V, Ingle M, Pandav N, Parikh P, Patel J, Phadke A, et al. The role of capsule endoscopy in etiological diagnosis and management of obscure gastrointestinal bleeding. Intest Res. 2016;14(1):69-74. https://doi.org/10.5217/ ir.2016.14.1.69.

9. Ersoy O, Harmanci O, Aydinli M, Sivri B, Bayraktar Y. Capability of capsule endoscopy in detecting small bowel ulcers. Dig Dis Sci. 2009;54(1):136-41. https://doi. org/10.1007/s10620-008-0320-0.

10. Cooper JE, Roberts-Thomson IC. Gastrointestinal: heterotopic gastric mucosa. J Gastroenterol Hepatol. 2001;16(4):475.

11. Terada T. Heterotopic gastric mucosa of the gastrointestinal tract: a histopathologic study of 158 cases. Pathol Res Pract. 2011;207(3):148-50. https://doi.org/10.1016/j. prp.2010.12.004.

12. Yu L, Yang Y, Cui L, Peng L, Sun G. Heterotopic gastric mucosa of the gastrointestinal tract: prevalence, histological features, and clinical characteristics. Scand J Gastroenterol. 2014;49(2):138-44. https://doi.org/10.3109/00365521.2 013.860558 .

13. Lee SM, Mosenthal WT, Weismann RE. Tumorous heterotopic gastric mucosa in the small intestine. Arch surgery. 1970;100(5):619-22. https://doi.org/10.1001/ archsurg.1970.01340230085022.

14. Qiao WG, Zhang LZ, Zhi FC. Tumor-like heterotopic gastric mucosa discovered by wireless capsule endoscopy. J Dig Dis. 2017;18(9):543-4. https://doi.org/10.1111/17512980.12464.
15. Tai CM, Chang IW, Wang HP. Heterotopic gastric mucosa of the ileum. Endoscopy. 2015;47(Suppl 1 UCTN):E423. https://doi.org/10.1055/s-0034-1392666.

16. Nawaz K, Graham DY, Fechner RE, Eiband JM. Gastric heterotopia in the ileum with ulceration and chronic bleeding. Gastroenterology. 1974;66(1):113-7. https://doi. org/10.1016/S0016-5085(74)80086-7.

17. Boybeyi O, Karnak I, Güçer S, Orhan D, Senocak ME. Common characteristics of jejunal heterotopic gastric tissue in children: a case report with review of the literature. J Pediatr Surg. 2008;43(7):e19-22. https://doi. org/10.1016/j.jpedsurg.2008.02.072.

18. Martínez A, Decanini-Terán O, Soria-Céspedes D. Polypoid and hyperplastic heterotopic gastric mucosa in the jejunum as a cause of recurrent subocclusive episodes. Ann Gastroenterol. 2013;26(2):184.

19. Abu-Zidan FM, El-Batrawy TM, Khan NH. Ectopic gastric mucosal ulcer of the jejunum without congenital anomaly causing intestinal obstruction. ANZ J Surg. 2018;88(12):E99-E100. https://doi.org/10.1111/ans.13318.

20. Chinnery GE, Bernon MM, Banderker MA, Roberts R, Krige JE. Gastric heterotopia causing jejunal ulceration and obstruction. S Afr J Surg. 2013;51(4):146-7. https://doi. org/10.7196/sajs.1735.

21. Houissa-Vuong S, Martin B, Lascar G, Vuong PN. Multiple jejunal strictures caused by gastric heterotopia. Ann Chir. 2001;126(1):70-4. https://doi.org/10.1016/S0003-3944 (00)00461-2.

22. Genta RM, Kinsey RS, Singhal A, Suterwala S. Gastric foveolar metaplasia and gastric heterotopia in the duodenum: no evidence of an etiologic role for Helicobacter pylori. Hum Pathol. 2010;41(11):1593-600. https://doi.org/10.1016/j. humpath.2010.04.010.

23. Kushima R, Borchard F, Hattori T. A new aspect of gastric metaplasia in Crohn's disease: bidirectional (foveolar and pyloric) differentiation in so-called 'pyloric metaplasia' in the ileum. Pathol Int. 1997;47(6):416-9. https://doi. org/10.1111/j.1440-1827.1997.tb04517.x.

24. Koukoulis GK, Ke Y, Henley JD, Cummings OW. Detection of pyloric metaplasia may improve the biopsy diagnosis of Crohn's ileitis. J Clin Gastroenterol. 2002;34(2):141-3. https://doi.org/10.1097/00004836-200202000-00007.

25. Agarwal S, Stucchi AF, Dendrinos K, Cerda S, O’Brien MJ, Becker JM, et al. Is pyloric gland metaplasia in ileal pouch biopsies a marker for Crohn's disease? Dig Dis Sci. 2013;58(10):2918-25. https://doi.org/10.1007/s10620013-2655-4. 